\title{
Treatment features of systemic chemotherapy in young adults with unresectable advanced or recurrent gastric cancer
}

This article was published in the following Dove Press journal: Cancer Management and Research

\section{Izuma Nakayama \\ Keisho Chin \\ Daisuke Takahari \\ Mariko Ogura \\ Takashi Ichimura \\ Takeru Wakatsuki \\ Hiroki Osumi \\ Yumiko Ota \\ Takeshi Suzuki \\ Mitsukuni Suenaga \\ Eiji Shinozaki \\ Kensei Yamaguchi}

Department of Gastroenterological Chemotherapy, Cancer Institute

Hospital of Japanese Foundation for

Cancer Research, Tokyo, Japan
Correspondence: Izuma Nakayama Department of Gastroenterological Chemotherapy, Cancer Institute Hospital of Japanese Foundation for Cancer Research, 3-8-3I Ariake, Koto-ku, Tokyo 135-8550, Japan

Tel +81335200111

Fax $+8 I 335700343$

Email izuma.nakayama@jfcr.or.jp
Purpose: Gastric cancer in young adults (GCYA) is known to have distinct clinicopathological features, including a female predominance and diffuse-type histology. Previous reports have focused on patients who had undergone gastrectomy with curative intent. Information concerning the treatment of unresectable advanced- or recurrent-stage GCYA is lacking. Therefore, we aimed to investigate whether the distinct clinicopathological features of GCYA affect the outcome of systemic chemotherapy.

Patients and methods: We conducted a retrospective cohort study at a single institution in Japan. GCYA was classified as a disease in individuals who were $<40$ years of age at diagnosis. Initial systemic chemotherapy regimens for GCYA were investigated with a focus on patients who received S-1 plus cisplatin (SP) as a representative standard regimen. The efficacy, safety, and feasibility of systemic chemotherapy were evaluated.

Results: Eighty-nine (7.5\%) of 1,184 consecutive patients who received systemic chemotherapy at our institute between December 2005 and June 2016 were enrolled. As reported previously, the female sex $(57.3 \%)$ and diffuse-type histology $(91.0 \%)$ were the dominant features of GCYA. Thirty-two patients $(36.0 \%)$ received SP as first-line treatment. The median overall survival and progression-free survival times were 13.2 (95.0\% CI: 9.5-18.7) and 5.6 (95.0\% CI: 4.7-7.9) months, respectively. The median number of treatment cycles, relative dose intensity, and cumulative dose of cisplatin were 4.5 (range: $1-10$ ), $92.0 \%$ (IQR: 83.5-98.3), and $286.5 \mathrm{mg} / \mathrm{m}^{2}$ (IQR: 172.5-367.5), respectively. The most common adverse event of Grade 3 or higher was neutropenia ( $n=5$ patients; $15.6 \%$ ). No patient had febrile neutropenia. Non-hematological adverse events of Grade 3 or higher were only observed in $2(6.3 \%)$ of 32 patients.

Conclusion: Standard chemotherapy used for general-aged GC patients has similar efficacy, reduced toxicity, and higher intensity in GCYA patients.

Keywords: efficacy, S-1 plus cisplatin, younger patients

\section{Introduction}

Gastric cancer (GC) represents the third most frequent cause of cancer-related deaths worldwide. ${ }^{1}$ GC most commonly occurs in individuals aged 50-70 years..$^{2-7}$ Previous reports ${ }^{3-8}$ have suggested that $\sim 3.0 \%-8.0 \%$ of GC patients are diagnosed at a younger age. GC in young adults (GCYA) has consistently been reported ${ }^{2-10}$ to have distinct clinicopathological features, such as female predominance, diffuse-type histology, and peritoneal metastasis. These studies have mainly focused on patients who had undergone gastrectomy with curative intent. ${ }^{2-16}$ The authors assessed patient demographics or prognoses, and compared them with those of more elderly patients. ${ }^{2,4-16}$ However, to 
the best of our knowledge, the use of systemic chemotherapy for the treatment of advanced GCYA is yet to be evaluated.

Fluoropyrimidine-platinum combination chemotherapy has been regarded as the standard treatment regimen for unresectable advanced or recurrent GC, based on the results of randomized controlled trials. ${ }^{17,18}$ However, because of its rarity, even in pivotal clinical trials, GCYA only accounts for a minority of cases. Therefore, the efficacy of standard systemic chemotherapy for GCYA has not been adequately evaluated in clinical trials.

Comprehensive molecular analysis by The Cancer Genome Atlas (TCGA) suggested that there are four molecular subtypes of advanced GCs. ${ }^{19}$ Diffuse-type histology and tumors occurring at an earlier age are enriched in the genomically stable (GS) subtype. $R H O A$ and $C D H 1$ mutations have been identified as key molecular alterations of tumors of the GS subtype. ${ }^{19}$ Recent molecular analysis of $\mathrm{GCYA}^{20}$ has shown that there are significant differences in the frequency of $\mathrm{RHOA}$ and $\mathrm{CDH} 1$ mutations between young and elderly patients with diffuse-type GC. These findings might imply that GCYA has differences in the molecular background compared with GC in generally aged patients.

In this study, we investigated whether the distinct clinicopathological features of GCYA affect the outcome of systemic chemotherapy. First, we examined the initial and subsequent systemic chemotherapy regimens for GCYA. Second, focusing on patients who received S-1 plus cisplatin (SP) as a representative standard regimen, we evaluated the efficacy, safety, and feasibility of systemic chemotherapy.

\section{Patients and methods}

\section{Patients}

The medical records of patients who received systemic chemotherapy between December 2005 and April 2016 at the Cancer Institute Hospital of the Japanese Foundation for Cancer Research (Tokyo, Japan) were reviewed. Patients who had histologically or cytologically confirmed unresectable advanced or recurrent gastric adenocarcinoma were selected. GCYA was classified in individuals who were $<40$ years of age at diagnosis. Clinicopathological characteristics (including age, sex, histological appearance of the biopsy sample [intestinal- or diffuse-type], macroscopic appearance of the biopsy sample [Borrmann type], laboratory data at the start of the initial chemotherapy, a family history of GC, and the number of metastatic sites) were evaluated. All participants provided written informed consent, and all data were fully de-identified. The study approval was waived by the Institutional Review Board of Cancer Institute Hospital of Japanese Foundation for Cancer Research because of the retrospective nature of the study. This study was conducted in accordance with the Helsinki Declaration.

\section{Chemotherapy regimens}

Chemotherapy regimens were determined at the physician's discretion. The preferred regimen for first-line treatment has changed over time. Following the SPIRITS trial, ${ }^{17} \mathrm{SP}$ became the standard regimen for first-line treatment. In 2011, the ToGA trial ${ }^{21}$ demonstrated the efficacy of trastuzumab for treating HER2-positive GC. Since then, trastuzumab with capecitabine plus cisplatin has been administered to patients with HER2-positive GC. Recently, oxaliplatin has been approved for the treatment of unresectable advanced or recurrent GC in Japan. Since 2014, S-1 plus oxaliplatin (SOX) has commonly been used to treat patients with HER2-negative GC at our institution. The treatment schedule and dose were the same as those reported in pivotal clinical trials. ${ }^{17,18,21}$

\section{Statistical analyses}

Overall survival (OS) and progression-free survival (PFS) were estimated using the Kaplan-Meier method and compared using the log-rank test. The cutoff date was May 29, 2018. OS was calculated from the date of commencing chemotherapy to the date of death from any cause. PFS was calculated from the date of commencing chemotherapy to the first date of disease progression (determined by imaging or a clinical condition). For PFS, patients who were transferred to a different hospital for treatment and died without confirmation of disease progression were censored at the last documented evaluation. Multivariate analysis was performed using a Cox regression model. Covariates with a $P<0.20$ in the univariate analysis were included in the multivariate analysis. In patients with measurable lesions, the objective response rate (ORR) was calculated according to the Response Evaluation Criteria in Solid Tumors (version 1.1). ${ }^{22}$ Adverse events were graded according to the Common Terminology Criteria for Adverse Events (version 4.0). All statistical analyses were conducted using EZR (Saitama Medical Center, Jichi Medical University, Saitama, Japan), a graphical user interface for R (The R Foundation for Statistical Computing, Vienna, Austria). ${ }^{23} P<0.05$ was considered statistically significant.

\section{Results}

\section{Patient characteristics}

In total, 1,184 consecutive patients received systemic chemotherapy as first-line treatment for unresectable advanced or recurrent gastric adenocarcinoma at our institution between 
December 2005 and June 2016. Eighty-nine patients (7.5\%) met the inclusion criteria and were enrolled in the study. The clinicopathological characteristics of the patients are summarized in Table 1. As previously reported, ${ }^{2-16}$ the female sex $(57.3 \%)$ and diffuse-type histology $(91.0 \%)$ were the dominant features of GCYA. Fifty-five patients $(61.8 \%)$ had a family history of cancer in first- or second-degree relatives, and 19 patients $(21.3 \%)$ had a family history of GC. Fortytwo patients (47.2\%) had Borrmann type 4 GC.

\section{First-line chemotherapy}

Two patients relapsed during adjuvant chemotherapy. Therefore, 87 patients were analyzed to determine the efficacy of first-line chemotherapy for GCYA. Fifty-eight patients (66.7\%) received fluoropyrimidine-platinum combination chemotherapy. The most commonly used regimen was SP $(n=35)$. Eight patients received capecitabine and cisplatin

Table I Baseline characteristics of the patients $(n=89)$

\begin{tabular}{|c|c|}
\hline Characteristic & n (\%) \\
\hline Median age (range), years & $35(16-39)$ \\
\hline \multicolumn{2}{|l|}{ Sex } \\
\hline Male & $38(42.7)$ \\
\hline Female & $51(57.3)$ \\
\hline \multicolumn{2}{|l|}{ Disease status } \\
\hline Unresectable & $74(83.1)$ \\
\hline Recurrent & $15(16.9)$ \\
\hline \multicolumn{2}{|l|}{ Family history of cancer } \\
\hline Yes / gastric cancer & $55(6 I .8) / 19(21.3)$ \\
\hline No & $21(23.6)$ \\
\hline Unknown & $13(14.6)$ \\
\hline \multicolumn{2}{|l|}{ ECOG performance status } \\
\hline 0 & $59(66.3)$ \\
\hline $\mathrm{I}$ & $23(25.8)$ \\
\hline 2 & $7(7.9)$ \\
\hline \multicolumn{2}{|l|}{ Histological appearance } \\
\hline Intestinal-type & $8(9.0)$ \\
\hline Diffuse-type & $81(91.0)$ \\
\hline \multicolumn{2}{|l|}{ Macroscopic appearance } \\
\hline Type 2 & $5(5.6)$ \\
\hline Type 3 & $29(32.6)$ \\
\hline Type 4 & $42(47.2)$ \\
\hline Others/unknown & $9(10.1) / 4(4.5)$ \\
\hline \multicolumn{2}{|l|}{ Primary tumor in place } \\
\hline Yes & $50(56.2)$ \\
\hline No & $39(43.8)$ \\
\hline \multicolumn{2}{|l|}{ Number of metastatic sites } \\
\hline $2>$ & $63(70.8)$ \\
\hline$\geq 2$ & $26(29.2)$ \\
\hline \multicolumn{2}{|l|}{ Adjuvant chemotherapy } \\
\hline Yes & $10(11.2)$ \\
\hline No & $79(88.8)$ \\
\hline
\end{tabular}

Abbreviation: ECOG, Eastern Cooperative Oncology Group. as the mainstay cytotoxic chemotherapy with molecular targeted agents. Other combinations included: $\operatorname{SOX}(n=6)$; irinotecan and cisplatin $(n=4)$; fluorouracil, leucovorin, and oxaliplatin ( $\mathrm{n}=2)$; docetaxel, cisplatin, and S-1 $(\mathrm{n}=1)$; and S-1 and irinotecan $(n=1)$. Thirty patients $(34.5 \%)$ were treated with single-agent chemotherapy. Seven of the 30 patients who received monotherapy started treatment before the results of the SPIRITS trial ${ }^{17}$ were published in 2007. Since SP has been regarded as the standard regimen for unresectable advanced or recurrent GC in Japan, patients intolerant to cisplatin and those having difficulty taking oral medication have only been treated with single agents, such as S-1 monotherapy $(n=12)$, methotrexate and fluorouracil $(n=4)$, fluorouracil plus folinic acid $(\mathrm{n}=3)$, and weekly paclitaxel (wPTX) (n=3) (Table 2).

All patients had discontinued first-line treatment by the cutoff date, mainly due to disease progression $(n=70$; $80.5 \%)$. Of the 17 patients (19.5\%) who discontinued firstline treatment without disease progression, three underwent conversion surgery, six requested treatment at another hospital before disease progression, and one initially treated with fluorouracil plus folinic acid switched to SP because of improved oral intake. Five patients completed first-line treatment with no detectable lesions on imaging after a longterm stable disease state. Two patients discontinued first-line treatment due to severe adverse events.

\section{Efficacy of SP for GCYA}

Among the 58 patients who received platinum-doublet therapy, 35 received SP as first-line treatment. Patients

Table 2 Chemotherapy regimens $(n=87)$

\begin{tabular}{|l|l|}
\hline Regimens & $\mathbf{n}(\%)$ \\
\hline Doublet chemotherapy & $58(66.7)$ \\
\hline SP & $35(40.2)$ \\
\hline XP & $8(9.2)$ \\
\hline SOX & $7(8.0)$ \\
\hline Irinotecan plus cisplatin & $4(4.6)$ \\
\hline mFOLFOX6 & $2(2.3)$ \\
\hline DCS & $\mathrm{I}(\mathrm{I} .1)$ \\
\hline S-I plus irinotecan & $\mathrm{I}(\mathrm{I} .1)$ \\
\hline Monotherapy & $29(33.3)$ \\
\hline S-I & $17(19.5)$ \\
\hline MTX/FU & $6(6.9)$ \\
\hline FL & $3(3.4)$ \\
\hline wPTX & $3(3.4)$ \\
\hline
\end{tabular}

Abbreviations: DCS, docetaxel, cisplatin, and S-I; FL, fluorouracil and leucovorin; FU, fluorouracil; mFOLFOX6, fluorouracil, leucovorin, and oxaliplatin; MTX, methotrexate; SOX, S-I plus oxaliplatin; SP, S-I plus cisplatin; wPTX, weekly paclitaxel; XP, capecitabine plus cisplatin. 
who underwent $\mathrm{R} 1$ resection for positive cytology and R0 metastasectomy $(n=3)$ were excluded. The patient demographics are summarized in Table 3. More patients with good physical conditions were included in the SP group than in the entire cohort. At the data cutoff date of May 29, 2018, all the patients were confirmed to be dead. The median follow-up was 13.2 months. The median OS and PFS were 13.2 (95.0\% CI: 9.5-18.7) (Figure 1) and 5.6 (95.0\% CI: 4.7-7.9) months (Figure 2), respectively. The 1- and 5-year OS rates were $59.4 \%$ (95.0\% CI: 40.5-74.0) and $3.1 \%$ (95.0\% CI: 0.2-13.7), respectively. No significant differences in OS were observed between patients receiving SP and other platinum-doublet regimens (log-rank test, $P=0.448$ ). However, there was a statistically significant difference in OS between patients treated with combination therapy (13.6 months, 95.0\% CI: 11.7-18.7) and monotherapy (6.5 months, 95.0\% CI: 4.1-7.4) (log-rank test, $P<0.001)$. The ORR of the 15 patients with measurable lesions was $46.7 \%$. In contrast, there was a significant difference in PFS between patients with Borrmann type 4 tumors and those with other types

Table 3 Demographics of patients treated with SP $(n=32)$

\begin{tabular}{|c|c|}
\hline Characteristic & n (\%) \\
\hline Median age (range), years & $35(16-39)$ \\
\hline \multicolumn{2}{|l|}{ Sex } \\
\hline Male & $13(40.6)$ \\
\hline Female & $19(59.4)$ \\
\hline \multicolumn{2}{|l|}{ Disease status } \\
\hline Unresectable & $25(78.1)$ \\
\hline Recurrent & $7(21.9)$ \\
\hline \multicolumn{2}{|l|}{ ECOG performance status } \\
\hline 0 & $27(84.4)$ \\
\hline 1 & $4(12.5)$ \\
\hline 2 & $\mathrm{I}(3 . \mathrm{I})$ \\
\hline \multicolumn{2}{|l|}{ Histological appearance } \\
\hline Intestinal-type & $\mathrm{I}(3 . \mathrm{I})$ \\
\hline Diffuse-type & $31(96.9)$ \\
\hline \multicolumn{2}{|l|}{ Macroscopic appearance } \\
\hline Type 2 & $2(6.3)$ \\
\hline Type 3 & $10(3 \mid .2)$ \\
\hline Type 4 & $16(50.0)$ \\
\hline Others/unknown & $4(12.5)$ \\
\hline \multicolumn{2}{|l|}{ Primary tumor in place } \\
\hline Yes & $17(53.1)$ \\
\hline No & $15(46.9)$ \\
\hline \multicolumn{2}{|l|}{ Number of metastatic sites } \\
\hline $2>$ & $20(62.5)$ \\
\hline$\geq 2$ & $12(37.5)$ \\
\hline \multicolumn{2}{|l|}{ Adjuvant chemotherapy } \\
\hline Yes & $4(12.5)$ \\
\hline No & $28(87.5)$ \\
\hline
\end{tabular}

Abbreviations: ECOG, Eastern Cooperative Oncology Group; SP, S-I plus cisplatin. of tumor (log-rank test, $P=0.024$ ) (Figure 3). Multivariate analysis revealed that patients with Borrmann type 4 tumors exhibited a trend toward longer PFS (HR: 0.41, 95.0\% CI: 0.17-1.01) and better OS (HR: 0.48, 95.0\% CI: 0.21-1.01) (Table 4).

\section{Feasibility and tolerability of SP for GCYA}

The median number of treatment cycles, relative dose intensity, and cumulative dose of cisplatin in patients treated with SP were 4.5 (range: 1-10), 92.0\% IQR: 83.5-98.3), and $286.5 \mathrm{mg} / \mathrm{m}^{2}$ (IQR: 172.5-367.5), respectively. The dose of S-1 was reduced in nine patients and that of cisplatin was reduced in eight patients. Approximately two-thirds of

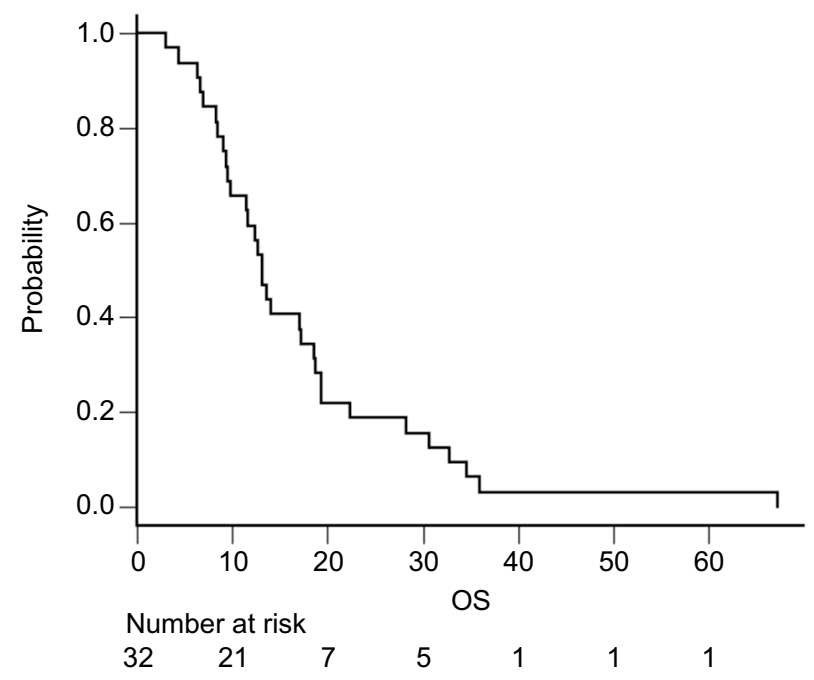

Figure I Kaplan-Meier curves of OS for patients who received SP ( $n=32)$. Abbreviations: OS, overall survival; SP, S-I plus cisplatin.

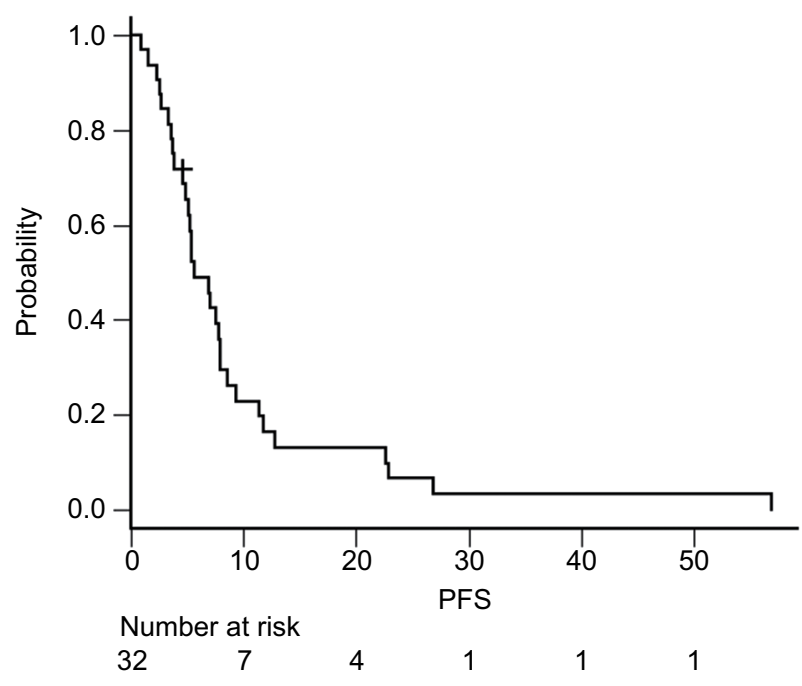

Figure 2 Kaplan-Meier curves of PFS for patients who received SP ( $n=32)$. Abbreviations: PFS, progression-free survival; SP, S-I plus cisplatin. 
patients $(n=21)$ did not require a reduction in $S-1$ or cisplatin dose throughout their entire treatment course. Additionally, $>40.0 \%$ of patients $(n=13)$ with adequate oral intake could be managed as outpatients.

Adverse events are summarized in Table 5. The most common adverse event of Grade 3 or higher was neutropenia $(\mathrm{n}=5 ; 15.6 \%)$, although no patient had febrile neutropenia. Non-hematological adverse events of Grade 3 or higher were only observed in $2(6.3 \%)$ of 32 patients. No patient required unscheduled hospitalization due to adverse events. There were no treatment-related deaths.

\section{Subsequent chemotherapy}

Of the 89 patients, 8 were transferred to another hospital during first-line chemotherapy and a further 8 did not

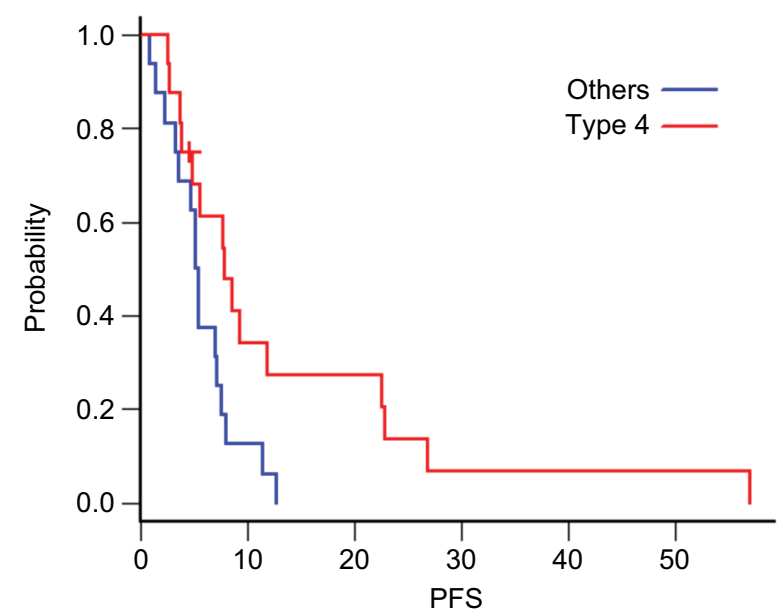

Number at risk

$\begin{array}{lllllll}\text { Others } & 16 & 2 & 0 & 0 & 0 & 0 \\ \text { Type } 4 & 16 & 5 & 4 & 1 & 1 & 1\end{array}$

Figure 3 Kaplan-Meier curves of PFS for patients with (red line) and without (blue lines) Borrmann type 4 tumors.

Abbreviation: PFS, progression-free survival. require subsequent chemotherapy ( 3 underwent conversion surgery; the remaining 5 had no evaluable disease after first-line treatment). Among the 73 patients who required subsequent chemotherapy, $54(74.0 \%)$ actually received second-line chemotherapy (taxane-based regimen $[n=37$; $68.5 \%$; ; irinotecan-based regimen [ $\mathrm{n}=10 ; 18.5 \%]$ ), and 28 (38.4\%) received third-line chemotherapy. The median OS and PFS of patients who received a taxane-based regimen were 5.2 (95.0\% CI: 4.1-6.4) and 1.8 (95.0\% CI: 1.6-2.3) months, respectively. The ORR of the 19 patients with measurable lesions was $5.3 \%$ (complete response [n=0],

Table 5 Adverse events $(n=32)$

\begin{tabular}{|l|l|l|}
\hline Adverse event & $\begin{array}{l}\text { All grades, } \\
\mathbf{n}(\%)\end{array}$ & $\begin{array}{l}\text { Grade } \\
\mathbf{3 / 4}, \mathbf{n}(\%)\end{array}$ \\
\hline Total & $32(100.0)$ & $10(31.3)$ \\
\hline Hematologic & $28(87.5)$ & $8(25.0)$ \\
Leukopenia & $18(56.3)$ & $0(0.0)$ \\
\hline Neutropenia & $14(43.8)$ & $5(15.6)$ \\
\hline Anemia & $21(61.5)$ & $3(9.4)$ \\
\hline Thrombocytopenia & $5(15.6)$ & $0(0.0)$ \\
\hline Total bilirubin increased & $13(40.6)$ & $0(0.0)$ \\
\hline AST/ALT increased & $12(37.5)$ & $0(0.0)$ \\
\hline ALP increased & $5(15.6)$ & $0(0.0)$ \\
\hline Creatinine increased & $3(9.4)$ & $0(0.0)$ \\
\hline Protein urea & $0(0.0)$ & $0(0.0)$ \\
\hline Febrile neutropenia & $0(0.0)$ & $0(0.0)$ \\
\hline Non-hematologic & $29(90.6)$ & $2(6.3)$ \\
\hline Anorexia & $23(71.9)$ & $0(0.0)$ \\
\hline Nausea & $20(62.5)$ & $0(0.0)$ \\
\hline Vomiting & $9(28.1)$ & $0(0.0)$ \\
\hline Fatigue & $18(56.3)$ & $0(0.0)$ \\
\hline Constipation & $12(37.5)$ & $0(0.0)$ \\
\hline Diarrhea & $18(56.3)$ & $2(6.3)$ \\
\hline Abreviations: ALP, & \\
\hline
\end{tabular}

Abbreviations: ALP, alkaline phosphatase; ALT, alanine aminotransferase; AST, aspartate aminotransferase.

Table 4 Multivariate analysis of OS and PFS $(n=32)$

\begin{tabular}{|c|c|c|c|c|}
\hline & OS & & PFS & \\
\hline Variable & HR (95\% Cl) & $P$-value & HR (95\% Cl) & $P$-value \\
\hline \multicolumn{5}{|c|}{ Borrmann Type 4} \\
\hline no & 1.00 & $P=0.070$ & 1.00 & $P=0.052$ \\
\hline yes & $0.48(0.21-1.06)$ & & $0.4 \mathrm{I}(0.17-1.0 \mathrm{I})$ & \\
\hline \multicolumn{5}{|c|}{ Serum CA 19-9 level } \\
\hline ULN> & 1.00 & $P=0.233$ & 1.00 & $P=0.345$ \\
\hline$\geq$ ULN & $1.68(0.7 \mathrm{I}-3.96)$ & & $\mathrm{I} .58(0.6 \mathrm{I}-4.08)$ & \\
\hline \multicolumn{5}{|c|}{ Serum CEA level } \\
\hline ULN> & 1.00 & $P=0.284$ & - & \\
\hline$\geq$ ULN & I.87 (0.60-5.86) & & - & \\
\hline
\end{tabular}

Note: “-” indicates serum CEA level was not significant for PFS in the univariate analysis. The multivariate analysis with this variable could not be performed. Abbreviations: CAI9-9, cancer antigen 19-9; CEA, carcinoembryonic antigen; OS, overall survival; PFS, progression-free survival; ULN, upper limit of normal. 
partial response $[n=1]$, stable disease $[n=6]$, progressive disease $[n=6]$, and not evaluable $[n=6])$.

\section{Discussion}

GCYA has not been clearly defined. Different definitions of GCYA have been adopted by each trial. Some studies ${ }^{2,4,5,7,9-16}$ have suggested that GCYA applies to patients $<40$ years of age, while others ${ }^{3,6,8}$ have suggested that it includes patients $\leq 45$ years of age. A younger age criterion has also been suggested..$^{13}$ The male-to-female ratio, a distinct feature of GCYA, gradually changes from a female to a male predominance at $\sim 40-45$ years of age. ${ }^{24}$ For that reason, we defined GCYA as tumors diagnosed at $<40$ years of age, thus enriching the patient population with distinct features of GCYA.

To the best of our knowledge, this is the first report evaluating the use of systemic chemotherapy for advanced GCYA. We have shown that approximately two-thirds of GCYA patients can receive platinum-doublet chemotherapy and that the clinical outcomes (OS, 13.2 months; PFS, 5.6 months; and ORR, $46.7 \%$ ) are comparable to those of pivotal clinical trials. ${ }^{17,18}$ Standard chemotherapy for the general-aged GC patients may have similar efficacy for GCYA patients. With regard to the safety profile of the SP regimen, both severe adverse events and those of any grade were less frequently observed in young adults compared with previously reported results in the general population. ${ }^{17,18}$ Twenty-two patients (68.7\%) did not experience any kind of severe adverse event. In the present study, the most common adverse event of Grade 3 or higher was neutropenia (15.6\%) (less than half of the rates reported in pivotal clinical trials [SPIRITS, 40.0\%; G-SOX, 41.8\%]). The favorable general conditions and organ function of young adults may facilitate intensive treatment. Considering their physical advantages, the similar survival outcome to the general population was not satisfactory for us. In a subgroup analysis of the G-SOX study of elderly patients $(n=99)$ aged $\geq 70$ years, ${ }^{25}$ the median number of treatment cycles, total dose, and relative dose intensity of cisplatin were 4.0 (IQR: $2.0-6.0$ ), $180.0 \mathrm{mg} / \mathrm{m}^{2}$, and $79.0 \%$, respectively. The median OS and PFS were $13.5(95.0 \% \mathrm{CI}$ : 11.2-17.9) and 5.5 (95.0\% CI: 4.1-6.7) months, respectively. The treatment intensity of this study ( 4.5 courses, $286.5 \mathrm{mg} /$ $\mathrm{m}^{2}$, and $92.0 \%$, respectively) was considerably higher than that of the G-SOX study. However, no significant differences in clinical outcomes were observed (OS, 13.2 months; PFS, 5.6 months). These findings suggest that GCYA may have a more aggressive tumor biology. $\mathrm{CDH} 1$ mutation could be a candidate gene alteration, resulting in tumor malignancy in GCYA patients. Comprehensive molecular analysis of dif- fuse-type GC demonstrated that the frequency of the somatic CDH1 mutation was higher in the early onset diffuse-type GC than in the late onset type. ${ }^{20}$ Somatic CDH1 structural mutation is recognized as a prognosis factor in sporadic GC. However, patients with a CDH1 structural mutation more frequently had an intestinal-type histology than patients with an epigenetic mutation. ${ }^{26}$ The combined data are insufficient to conclude that only $\mathrm{CDH} 1$ mutation may be responsible for the aggressiveness of GCYA. Further molecular analysis is therefore warranted. According to the TCGA analysis, microsatellite instability-high (MSI-H) tumors were enriched in elderly patients. As MSI-H GC is known to have a better prognosis, ${ }^{27}$ this may be the reason for the relatively aggressive tumor biology of GCYA.

Subsequent treatment outcomes after first-line chemotherapy were worse than those of the general GC populations included in clinical trials. ${ }^{28-30}$ The efficacy of taxane-based regimens in the present study (OS, 5.2 months; PFS, 1.8 months; and ORR, 5.3\%) was poor and the proportion of patients receiving third-line chemotherapy was relatively low (38.4\%) compared with the proportion of patients who were treated with wPTX in pivotal Japanese clinical trials. ${ }^{28-30}$ Patients with diffuse-type GC tend to have peritoneal metastasis and it is difficult to evaluate peritoneal metastasis by CT. Most patients had ascites or some clinical symptoms caused by peritoneal metastasis after first-line chemotherapy. The patients' general condition at the start of second-line chemotherapy may affect the outcome of the subsequent chemotherapy. Early detection and control of tumor progression are important for improving the outcome of GCYA. Ramucirumab plus nab-paclitaxel is expected to be a promising treatment regimen for GCYA. Recently, published Phase II data ${ }^{31}$ have shown that ramucirumab plus nab-paclitaxel produces a favorable ORR (54.8\%) in the second-line treatment of advanced GC. However, the incidence of Grade 3 or higher neutropenia was exceedingly high (76.7\%). As mentioned previously, GCYA patients are likely to have good bone marrow function and could tolerate this intensive regimen. Furthermore, in a subgroup analysis of the ABSOLUTE trial, ${ }^{32}$ it has been reported that nab-paclitaxel has greater efficacy for peritoneal metastasis than paclitaxel. Most GCYA patients had diffuse-type histology. Therefore, disease control of peritoneal metastasis is important for improving outcomes after progression.

This study had several limitations. First, this study was conducted at a single Japanese institution. Owing to the small sample size, it was difficult to draw definitive conclusions from the data. However, considering the rarity of GCYA, 
this was a relatively large-scale study. Furthermore, the clinical outcomes of the patients in this study are consistent with those of previous studies of SP. ${ }^{17,18}$ Second, the clinical outcomes of patients in pivotal clinical trials were used as the control data. In general, patients enrolled in clinical trials represent a selective population with good major organ function and few complications. Patients, especially those treated with SP, may be comparable to those enrolled in clinical trials. Third, trastuzumab is the standard molecular targeting agent for HER2-positive advanced GC. The HER2 status of 17 patients $(53.1 \%)$ was not examined because first-line chemotherapy was started before the approval of trastuzumab. However, considering the histology of GCYA, few HER2positive tumors were expected in our cohort. In fact, all 15 patients who were examined had a negative HER2 status. Finally, all patients in this study were Japanese and mainly received S-1-based chemotherapy. There are several differences in the treatment strategy, surgical method, cancer stage, etiology, and clinical outcome between GC in eastern and western countries. ${ }^{33}$ Moreover, some investigators showed that GC in western countries is biologically different from that in eastern countries. ${ }^{34}$ Interestingly, however, the unique clinicopathological features of GCYA in sex and histology were commonly reported in both eastern ${ }^{2,4-7,9,10,12}$ and western countries. ${ }^{3,8,11}$ A comprehensive molecular analysis of GCYA was recently published from South Korea. ${ }^{20}$ In this study, there were only two patients $<40$ years of age in the TCGA stomach cohort and they were from Vietnam. Thus, the molecular background of GCYA in western countries remains unknown. Furthermore, $\mathrm{S}-1$ is one of the key agents for the treatment of GC in East Asia but is not used globally. In our study, there was no significant difference in the clinical outcome between patients treated with SP and other combination chemotherapies. Therefore, our results could be, to some extent, applicable for GCYA patients outside of Japan.

According to the analysis of TCGA GC data, GS tumors represent the most common type of GCYA. GS tumors have few actionable genomic features, such as driver mutations, copy number alterations of receptor tyrosine kinases, and mutation burden. ${ }^{19}$ GS tumors are not considered to be good candidates for existing molecularly targeted agents or immune checkpoint inhibitors. Different approaches are needed to develop a novel target therapy for GCYA.

\section{Conclusion}

Standard chemotherapy for general-aged GC patients has similar efficacy, reduced toxicity, and higher intensity for GCYA.

\section{Abbreviations}

CI, confidence interval; GC, gastric cancer; GCYA, gastric cancer in young adults; GS, genetically stable; IQR, interquartile range; ORR, objective response rate; OS, overall survival; PFS, progression-free survival; SP, S-1 plus cisplatin.

\section{Acknowledgments}

The authors thank the staff who managed the patients at the Ambulatory Treatment Center and in the ward. The authors also thank Editage for English language editing.

\section{Disclosure}

TI has received lecture fees from Eli Lilly Japan KK (Hyogo, Japan) and has received research grants from Clinico Co Ltd (Tokyo, Japan). ES has received lecture fees from Merck Serono Co Ltd (Tokyo, Japan), Takeda Pharmaceutical Co Ltd (Osaka, Japan), Chugai Pharmaceutical Co Ltd (Tokyo, Japan), Taiho Pharmaceutical Co Ltd (Tokyo, Japan), Bayer Yakuhin Ltd (Osaka, Japan), and Yakult Honsha Co Ltd (Tokyo, Japan) and has had acceptance of researchers from Toppan Printing Co Ltd (Tokyo, Japan). KY has received lecture fees from Eli Lilly Japan KK (Hyogo, Japan), Merck Serono Co Ltd (Tokyo, Japan), Takeda Pharmaceutical Co Ltd (Osaka, Japan), Chugai Pharmaceutical Co Ltd (Tokyo, Japan), Taiho Pharmaceutical Co Ltd (Tokyo, Japan), and Yakult Honsha Co Ltd (Tokyo, Japan) and has received research grants from Taiho Pharmaceutical Co Ltd (Tokyo, Japan) and Yakult Honsha Co Ltd (Tokyo, Japan). The other authors report no conflicts of interest in this work.

\section{References}

1. World Health Organization. International Agency for Research on Cancer. GLOBOCAN 2012: Estimated Cancer Incidence, Mortality and Prevalence Worldwide in 2012 v1.0; 2012. Available from: http://globocan.iarc. fr/Pages/fact_sheets_population.aspx Accessed August 1, 2016.

2. Katai H, Sasako M, Sano T, Maruyama K. Gastric carcinoma in young adults. Jpn J Clin Oncol. 1996;26(3):139-143.

3. Al-Refaie WB, Hu CY, Pisters PW, Chang GJ. Gastric adenocarcinoma in young patients: a population-based appraisal. Ann Surg Oncol. 2011;18(10):2800-2807.

4. Takatsu Y, Hiki N, Nunobe S, et al. Clinicopathological features of gastric cancer in young patients. Gastric Cancer. 2016;19(2):472-478.

5. Liu S, Feng F, Xu G, et al. Clinicopathological features and prognosis of gastric cancer in young patients. BMC Cancer. 2016;16:478.

6. Qiu MZ, Wang ZQ, Zhang DS, et al. Clinicopathological characteristics and prognostic analysis of gastric cancer in the young adult in China. Tumour Biol. 2011;32(3):509-514.

7. Dhobi MA, Wani KA, Parray FQ, et al. Gastric cancer in young patients. Int J Surg Oncol. 2013;2013:981654.

8. Santoro R, Carboni F, Lepiane P, Ettorre GM, Santoro E. Clinicopathological features and prognosis of gastric cancer in young European adults. Br J Surg. 2007;94(6):737-742. 
9. Park JC, Lee YC, Kim JH, et al. Clinicopathological aspects and prognostic value with respect to age: an analysis of 3,362 consecutive gastric cancer patients. J Surg Oncol. 2009;99(7):395-401.

10. Hsieh FJ, Wang YC, Hsu JT, Liu KH, Yeh CN. Clinicopathological features and prognostic factors of gastric cancer patients aged 40 years or younger. J Surg Oncol. 2012;105(3):304-309.

11. Koea JB, Karpeh MS, Brennan MF. Gastric cancer in young patients: demographic, clinicopathological, and prognostic factors in 92 patients. Ann Surg Oncol. 2000;7(5):346-351.

12. Maehara Y, EmiY, Tomisaki S, et al. Age-related characteristics of gastric carcinoma in young and elderly patients. Cancer. 1996;77(9):1774-1780.

13. Kim DY, Joo JK, Ryu SY, Park YK, Kim YJ, Kim SK. Clinicopathologic characteristics of gastric carcinoma in elderly patients: a comparison with young patients. World J Gastroenterol. 2005;11(1):22-26.

14. Choi JH, Chung HC, Yoo NC, et al. Gastric cancer in young patients who underwent curative resection. Comparative study with older patients. Am J Clin Oncol. 1996;19(1):45-48.

15. Nakamura R, Saikawa Y, Takahashi T, et al. Retrospective analysis of prognostic outcome of gastric cancer in young patients. Int J Clin Oncol. 2011;16(4):328-334.

16. Isobe T, Hashimoto K, Kizaki J, et al. Characteristics and prognosis of gastric cancer in young patients. Oncol Rep. 2013;30(1):43-49.

17. Koizumi W, Narahara H, Hara T, et al. S-1 plus cisplatin versus S-1 alone for first-line treatment of advanced gastric cancer (SPIRITS trial): a phase III trial. Lancet Oncol. 2008;9(3):215-221.

18. Yamada Y, Higuchi K, Nishikawa K, et al. Phase III study comparing oxaliplatin plus S-1 with cisplatin plus S-1 in chemotherapy-naïve patients with advanced gastric cancer. Ann Oncol. 2015;26(1):141-148.

19. Cancer Genome Atlas Research Network. Comprehensive molecular characterization of gastric adenocarcinoma. Nature. 2014;513(7517):202-209.

20. Cho SY, Park JW, Liu Y, et al. Sporadic early-onset diffuse gastric cancers have high frequency of somatic $C D H 1$ alterations, but low frequency of somatic RHOA mutations compared with late-onset cancers. Gastroenterology. 2017;153(2):536-549.

21. Bang YJ, van Cutsem E, Feyereislova A, et al. Trastuzumab in combination with chemotherapy versus chemotherapy alone for treatment of HER2-positive advanced gastric or gastro-oesophageal junction cancer (ToGA): a phase 3, open-label, randomised controlled trial. Lancet. 2010;376(9742):687-697.

22. Eisenhauer EA, Therasse P, Bogaerts J, et al. New response evaluation criteria in solid tumours: revised RECIST guideline (version 1.1). Eur $J$ Cancer. 2009;45(2):228-247.
23. Kanda Y. Investigation of the freely available easy-to-use software 'EZR' for medical statistics. Bone Marrow Transplant. 2013;48(3):452-458.

24. Yu J, He Y, Guo Z. Age trend of the male to female sex ratio in surgical gastric cancer patients at a single institution. World J Surg Oncol. 2014;12:269-272.

25. Bando H, Yamada Y, Tanabe S, et al. Efficacy and safety of S-1 and oxaliplatin combination therapy in elderly patients with advanced gastric cancer. Gastric Cancer. 2016;19(3):919-926.

26. Corso G, Carvalho J, Marrelli D, et al. Somatic mutations and deletions of the E-cadherin gene predict poor survival of patients with gastric cancer. J Clin Oncol. 2013;31(7):868-875.

27. Zhu L, Li Z, Wang Y, et al. Microsatellite instability and survival in gastric cancer: a systematic review and meta-analysis. Mol Clin Oncol. 2015;3(3):699-705.

28. Hironaka S, Ueda S, Yasui H, et al. Randomized, open-label, phase III study comparing irinotecan with paclitaxel in patients with advanced gastric cancer without severe peritoneal metastasis after failure of prior combination chemotherapy using fluoropyrimidine plus platinum: WJOG 4007 trial. J Clin Oncol. 2013;31(35):4438-4444.

29. Wilke H, Muro K, van Cutsem E, et al. Ramucirumab plus paclitaxel versus placebo plus paclitaxel in patients with previously treated advanced gastric or gastro-oesophageal junction adenocarcinoma (RAINBOW): a double-blind, randomised phase 3 trial. Lancet Oncol. 2014;15(11): 1224-1235.

30. Shitara K, Takashima A, Fujitani K, et al. Nab-paclitaxel versus solventbased paclitaxel in patients with previously treated advanced gastric cancer (ABSOLUTE): an open-label, randomised, non-inferiority, phase 3 trial. Lancet Gastroenterol Hepatol. 2017;2(4):277-287.

31. Bando H, Shimodaira H, Fujitani K, et al. A phase II study of nabpaclitaxel in combination with ramucirumab in patients with previously treated advanced gastric cancer. Eur J Cancer. 2018;91:86-91.

32. Takashima A, Shitara K, Fujitani K, et al. Peritoneal metastasis as a predictive factor for nab-paclitaxel in patients with pretreated advanced gastric cancer: an exploratory analysis of the phase III ABSOLUTE trial. Gastric Cancer. 2018.

33. Yamamoto M, Rashid OM, Wong J. Surgical management of gastric cancer: the East vs. West perspective. J Gastrointest Oncol. 2015;6(1): 79-88.

34. Markar SR, Karthikesalingam A, Jackson D, Hanna GB. Long-term survival after gastrectomy for cancer in randomized, controlled oncological trials: comparison between West and East. Ann Surg Oncol. $2013 ; 20(7): 2328-2338$
Cancer Management and Research

\section{Publish your work in this journal}

Cancer Management and Research is an international, peer-reviewed open access journal focusing on cancer research and the optimal use of preventative and integrated treatment interventions to achieve improved outcomes, enhanced survival and quality of life for the cancer patient. The manuscript management system is completely online and includes

\section{Dovepress}

a very quick and fair peer-review system, which is all easy to use. Visi $\mathrm{http}: / /$ www.dovepress.com/testimonials.php to read real quotes from published authors. 